\title{
A Cytotoxic Compound from n-Hexane Fraction of Lantana camara Linn Leaves
}

\author{
Suryati ${ }^{*}$, Yuni Malasari', Mai Efdi', Elida Mardiah' \\ 'Department of Chemistry, Faculty of Mathematics and Science, Andalas University, Padang, Indonesia. \\ ${ }^{*}$ Corresponding author email: suryati@sci.unand.ac.id
}

Received: 21 Feb 2019; Accepted: 28 May 2019; Available online: 5 Jun 2019

\begin{abstract}
In this study, one triterpenoid compound from $n$-hexane fraction of Lantana camara Linn leaves has cytotoxic activity was isolated. Isolation was carried out using gravity chromatography column and purification by recrystallization method. Isolated compound obtained was white solid with melting point $252-253^{\circ} \mathrm{C}$. The structure of isolated compound was elucidated using spectroscopic analysis Ultraviolet (UV), Infrared (IR), ${ }^{1} \mathrm{H}-\mathrm{Nuclear}$ Magnetic Resonance $\left({ }^{1} \mathrm{H}-\mathrm{NMR}\right),{ }^{13} \mathrm{C}$ Nuclear Magnetic Resonance $\left({ }^{13} \mathrm{C}-\mathrm{NMR}\right)$, Heteronuclear Multiple Bond Connectivity (HMBC), Heteronuclear Multiple Quantum Correlation (HMQC), Distortionless Enhancement Polarization Transfer (DEPT) and comparative literature data, identified as Lantadene A (226-angeloyloxy-3-oxoolean-12-en-28-oic-acid) with molecule formula $\mathrm{C}_{35} \mathrm{H}_{52} \mathrm{O}_{5}$. The Lantadene A compound was evaluated for cytotoxic activity against shrimp larvae Artemia Salina Leach using Brine Shrimp Lethality Test (BSLT) method, showed strong cytotoxic activity with an $\mathrm{LC}_{50}$ value of $48.97 \mu \mathrm{g} / \mathrm{mL}$.
\end{abstract}

Keywords : Lantana camara Linn, Lantadene A, Artemia Salina Leach, Cytotoxic.

\section{INTRODUCTION}

Lantana camara Linn plant has various activities such as antibacterial (Ganjewala, Sam, \& Khan, 2009), anticancer (Pour, Latha \& Sashidaran, 2011), antifungal (Passos et al., 2012), anti-inflamatory, antiviral, antitubercular, nematicidal, hepatotoxic, antimutagenic (Kumar, Katiyar, Singh, Surender \& Tarun, 2016), antioxidant (Suryati, Santoni, M.Z, \& Aziz, 2016), antidiabetic (Kazmi et al., 2012), and cytotoxic activity (Ediruslan, Manjang, Suryati \& Aziz, 2015).

Several compounds have been isolated from Lantana camara Linn such as 9-Hydroxy-lantadene A (M.Z, Suryati \& Efdi, 2018), 24-Hydroxy-lantadene B, 24Hydroxy-lantadene X, 24-Hydroxy-lantadene D, 22Hydroxy-4-epi-hederagonic acid, $3 \beta$-Hydroxy-lantadene C (Abdjul, et al, 2017), ursolic acid (Jamal, Amir, Ali, \& Mujeeb, 2018), oleanolic acid (Vyas \& Argal, 2014), camaric acid, Lantadene $\mathrm{C}$, camarinic acid, camaraside, 24-Hydroxy-3-oxours-12-en-28-oic acid (Kumar, et al., 2016).

Previous research, Ediruslan et al. (2015) has reported the cytotoxic of n-hexane, and ethyl acetate fractions of Lantana camara Linn leaves. $n$-Hexane and ethyl acetate fractions showed strong toxicity with $\mathrm{LC}_{50}$ values of 34 and $27 \mu \mathrm{g} / \mathrm{mL}$, respectively. Ediruslan et al. (2015) has also reported triterpenoid compound (Lantanilic acid) show strong cytotoxic potential with $\mathrm{LC}_{50}$ values of $27.99 \mu \mathrm{g} / \mathrm{mL}$ of ethyl acetate, but not yet reported cytotoxic compound from n-hexane fraction of Lantana camara Linn leaves.

This paper reported the isolation of triterpenoid compound from n-hexane fraction of Lantana camara Linn leaves and its cytotoxic activity using Brine Shrimp Lethality Test (BSLT) method. The structure of isolated compound was elucidated using spectroscopic analysis UV, IR and NMR (1D and 2D).

\section{EXPERIMENTAL SECTION Material}

Lantana camara Linn leaves were collected from area of Limau Manis, Pauh sub-district, Padang city, West Sumatera province, Indonesia. This plant has been identified in the Herbarium Laboratory Andalas University (ANDA), Department of Biology, Faculty of Mathematics and Natural Sciences, Andalas University with the specimen code 472/ K-ID/ANDA/XII/2017.

Organic solvent such as $n$-hexane, ethyl acetate, methanol (Brataco) using distillate, Liebermann Burchard Reagent, Silica Gel 60 (Merck, 0.063-0.200 mm), Thin Layer Chromatography (TLC) (DC-Kiesegel $60 \begin{array}{lll}\mathrm{F}_{254} & \end{array}$ Merck), and cytotoxic activity test such as shrimp larvae eggs Artemia Salina Leach, seawater, dimethyl sulfoxide (DMSO).

\section{Apparatus}

UV spectra recorded with spectrophotometer (Thermo Scientific, Genesys 10 UV-Vis), FT-IR spectra measured with spectrophotometer (Perkin Elmer, Frontier), NMR spectra collected using spectrometer (ECX400). ${ }^{1}$ Hydrogen-Nuclear Magnetic Resonance ( ${ }^{1} \mathrm{H}-\mathrm{NMR}$, $\left.\mathrm{CDCl}_{3}\right)$ ECX400 (400 MHz), ${ }^{13}$ Carbon-Nuclear Magnetic Resonance $\left({ }^{13} \mathrm{C}-\mathrm{NMR}, \mathrm{CDCl}_{3}\right)$ ECX400 (100 MHz) with tetramethylsilane (TMS) as standard.

\section{Extraction, Isolation and Structure Determination}

Dry sample of Lantana camara Linn leaves (3 kg) macerated with methanol solvent. Maceration was performed repeatedly until the macerate obtained colorless. 
It was then filtered and concentrated with rotary evaporator to obtain crude methanolic extract $(400 \mathrm{~g})$. The crude methanolic extract (400 g) was partitioned with $n$-hexane and obtained $n$-hexane fraction $(40 \mathrm{~g})$.

$n$-Hexane fraction $(40 \mathrm{~g})$ was fractionated by gravity chromatography column (0.063-0.200 mesh) with hexane:ethyl acetate $(10: 0 ; 0: 10)$ and ethyl acetate:methanol $(10: 0 ; 0: 10)$ as gradient eluent and obtained 13 fractions $\left(F_{1}-F_{13}\right)$. Then, all fractions were evaluated for cytotoxic activity against shrimp larvae Artemia salina Leach. Fraction $\mathrm{F}_{13} \quad\left(\begin{array}{ll}3 & \mathrm{~g}\end{array}\right)$ was rechromatographed on a silica gel column using hexane:ethyl acetate (10:0;0:10) and ethyl acetate:methanol $(10: 0 ; 0: 10)$ as the eluent and obtained 10 sub-fractions $\left(F_{13.1}-F_{13.10}\right)$. Then, all sub-fractions were repeatedly evaluated cytotoxic activity against shrimp larvae Artemia salina Leach. Sub-fraction $\mathrm{F}_{13.3}(1 \mathrm{~g})$ was separated on silica gel column, eluted with $n$-hexane:ethyl acetate (10:0-0:10) and obtained 13 sub-fractions $\left(\mathrm{F}_{13.3 .1^{-}}\right.$

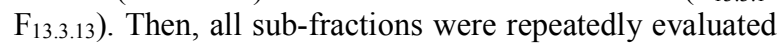
cytotoxic activity against shrimp larvae Artemia salina Leach using Brine Shrimp Lethality Test (BSLT). Subfraction $\mathrm{F}_{13.3 .4}$ was purified using recrystallization with hexane:ethyl acetate, obtained pure compound $(15 \mathrm{mg})$. Gave a triterpenoid positive test with Liebermann Burchard reagent and evaluated for cytotoxic activity. The isolated compound was carried out the melting point measurement and the structure was elucidated using spectroscopic analysis UV, IR, and NMR (1D and 2D).

\section{Cytotoxic Activity Test \\ Sample preparation}

The isolated compound ( $2 \mathrm{mg}$ ) was dissolved with $n$ hexane in $2 \mathrm{~mL}$ flask and obtained a stock solution (1000 $\mu \mathrm{g} / \mathrm{mL})$. Five different concentrations such as $250 ; 125$; $62.5 ; 31.25$ and $15.625 \mu \mathrm{g} / \mathrm{mL}$ were prepared in triplicate by dilution from a stock solution.

\section{Hatching of shrimp larvae Artemia salina Leach}

Seawater was put into a glass container such as the dark and light part. Shrimp larvae eggs Artemia salina Leach were put into the dark part of the container at temperature $24-26^{\circ} \mathrm{C}$ (Vajha \& Krishna, 2014). After 48 hours, the phototropic nauplii will move into the light part. These larvae were used as experimental animal in the cytotoxic test using Brine Shrimp Lethality Test (BSLT) method (Olowa \& Nuñeza, 2013).

\section{Brine Shrimp Lethality Test (BSLT)}

The isolated compound with different concentrations such as $250 ; 125 ; 62.5 ; 31.25$ and $15.625 \mu \mathrm{g} / \mathrm{mL}$ were put into vial and dried. Further, $50 \mu \mathrm{L}$ DMSO was added to each vial until homogeneous (Sivasankar et al., 2013). Then, ten shrimp larvae were put to each vials using pasteur pipette and added $5 \mathrm{~mL}$ of seawater in vial (Musa, 2012). Each vial contain shrimp larvae were placed in the light and after 24 hours shrimp larva Artemia salina Leach observed and calculated total of dead larvae in each vial. The percentage of mortality of shrimp larvae Artemia salina Leach on each concentration were determined using probit analysis. Lethality Concentration $\left(\mathrm{LC}_{50}\right)$ value determined using a regression equation.

\section{RESULTS AND DISCUSSION}

The result ( $40 \mathrm{~g}$ ) of $n$-hexane fraction was fractionated by silica gel gravity chromatography column and purification by recrystallization, obtained pure compound $\left(15 \mathrm{mg}\right.$ ) with melting point $252-253^{\circ} \mathrm{C}$. The structure of isolated compound was elucidated using spectroscopic analysis UV, IR, and NMR (1D and 2D) and identified by comparative literature data (Table 1).

The data value the chemical shift of triterpenoid isolated compound was compared by published Lantadene A data (Table 1), the chemical shift value of the isolation has high similarity with comparative Lantadene A data, from the comparative data identified as Lantadene A $(22 \beta-$ angeloyloxy-3-oxoolean-12-en-28-oic-acid) (Figure 1).

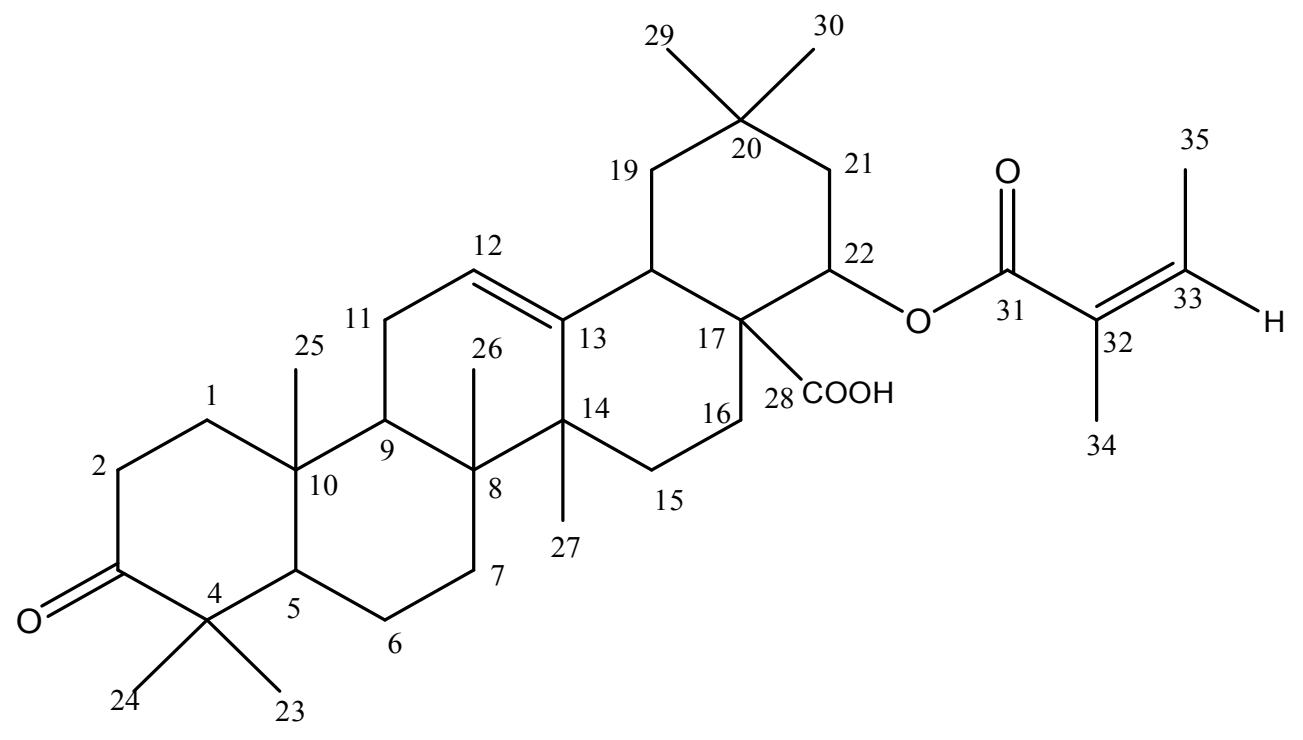

Figure 1. Chemical structure of Lantadene A (22ß-angeloyloxy-3-oxoolean-12-en-28-oic-acid) 
Table 1. ${ }^{1} \mathrm{H}-\mathrm{NMR}$ data $\left(400 \mathrm{MHz}, \mathrm{CDCl}_{3}\right)$ and ${ }^{13} \mathrm{C}-\mathrm{NMR}$ data $\left(100 \mathrm{MHz}, \mathrm{CDCl}_{3}\right)$ isolated compound and comparative Lantadene A data (Manu, Sharma, Bansal \& Singh, 2007).

\begin{tabular}{|c|c|c|c|c|c|}
\hline \multirow[b]{2}{*}{ No } & \multicolumn{4}{|c|}{ Isolated compound } & \multirow{2}{*}{$\begin{array}{l}\text { Literatur } \\
\delta \mathrm{c}(\mathrm{ppm})\end{array}$} \\
\hline & $\delta \mathrm{c}(\mathrm{ppm})$ & DEPT & HMQC & HMBC & \\
\hline 1 & 39.1 & $\mathrm{CH}_{2}$ & $1.5\left(\mathrm{H}_{1}\right)$ & $23.5\left(\mathrm{C}_{11}\right)$ & 38.4 \\
\hline 2 & 34.2 & $\mathrm{CH}_{2}$ & $2.3\left(\mathrm{H}_{2}\right)$ & $39.1\left(\mathrm{C}_{1}\right)$ & 34.1 \\
\hline 3 & 217.7 & $\mathrm{C}$ & & & 217.6 \\
\hline 4 & 47.5 & $\mathrm{C}$ & & & 47.4 \\
\hline 5 & 55.3 & $\mathrm{CH}$ & $1.3\left(\mathrm{H}_{5}\right)$ & $26.5\left(\mathrm{C}_{23}\right)$ & 55.2 \\
\hline 6 & 19.8 & $\mathrm{CH}_{2}$ & & & 19.5 \\
\hline 7 & 32.2 & $\mathrm{CH}_{2}$ & & & 32.1 \\
\hline 8 & 39.3 & $\mathrm{C}$ & & & 39.2 \\
\hline 9 & 46.9 & $\mathrm{CH}$ & & & 47.7 \\
\hline 10 & 36.8 & $\mathrm{C}$ & & & 36.7 \\
\hline 11 & 23.5 & $\mathrm{CH}_{2}$ & $1.2\left(\mathrm{H}_{11}\right)$ & $143.1\left(\mathrm{C}_{13}\right) ; 15.7\left(\mathrm{C}_{25}\right)$ & 23.5 \\
\hline 12 & 122.6 & $\mathrm{CH}$ & $5.3\left(\mathrm{H}_{12}\right)$ & $42.1\left(\mathrm{C}_{14}\right)$ & 122.4 \\
\hline 13 & 143.1 & $\mathrm{C}$ & & & 143.1 \\
\hline 14 & 42.1 & $\mathrm{C}$ & & & 41.9 \\
\hline 15 & 27.6 & $\mathrm{CH}_{2}$ & $1.0\left(\mathrm{H}_{15}\right)$ & $143.1\left(\mathrm{C}_{13}\right) ; 42.1\left(\mathrm{C}_{14}\right)$ & 27.5 \\
\hline 16 & 24.2 & $\mathrm{CH}_{2}$ & $0.9\left(\mathrm{H}_{16}\right)$ & $42.1\left(\mathrm{C}_{14}\right)$ & 24.1 \\
\hline 17 & 50.6 & $\mathrm{C}$ & & & 50.6 \\
\hline 18 & 38.6 & $\mathrm{CH}$ & $3.0\left(\mathrm{H}_{18}\right)$ & & 38.4 \\
\hline 19 & 46.0 & $\mathrm{CH}_{2}$ & $1.6\left(\mathrm{H}_{19}\right)$ & $38.6\left(\mathrm{C}_{18}\right)$ & 46.8 \\
\hline 20 & 30.1 & $\mathrm{C}$ & & & 30.0 \\
\hline 21 & 37.8 & $\mathrm{CH}_{2}$ & & & 37.7 \\
\hline 22 & 75.9 & $\mathrm{CH}$ & $5.0\left(\mathrm{H}_{22}\right)$ & $30.1\left(\mathrm{C}_{20}\right)$ & 75.8 \\
\hline 23 & 26.5 & $\mathrm{CH}_{3}$ & $1.2\left(\mathrm{H}_{23}\right)$ & & 26.4 \\
\hline 24 & 21.5 & $\mathrm{CH}_{3}$ & $1.1\left(\mathrm{H}_{24}\right)$ & $217.7\left(\mathrm{C}_{3}\right)$ & 21.4 \\
\hline 25 & 15.1 & $\mathrm{CH}_{3}$ & $1.0\left(\mathrm{H}_{25}\right)$ & & 15.0 \\
\hline 26 & 16.8 & $\mathrm{CH}_{3}$ & $0.8\left(\mathrm{H}_{26}\right)$ & $32.2\left(\mathrm{C}_{7}\right) ; 39.3\left(\mathrm{C}_{8}\right) ; 46.9\left(\mathrm{C}_{9}\right)$ & 16.8 \\
\hline 27 & 25.8 & $\mathrm{CH}_{3}$ & $0.9\left(\mathrm{H}_{27}\right)$ & & 25.7 \\
\hline 28 & 178.4 & $\mathrm{C}$ & & & 180.1 \\
\hline 29 & 33.7 & $\mathrm{CH}_{3}$ & $0.9\left(\mathrm{H}_{29}\right)$ & $46.0\left(\mathrm{C}_{19}\right) ; 30.1\left(\mathrm{C}_{20}\right) ; 37.8\left(\mathrm{C}_{21}\right) ; 26.2\left(\mathrm{C}_{30}\right)$ & 33.6 \\
\hline 30 & 26.2 & $\mathrm{CH}_{3}$ & $1.0\left(\mathrm{H}_{30}\right)$ & & 26.1 \\
\hline 31 & 166.3 & $\mathrm{C}$ & & & 166.2 \\
\hline 32 & 127.6 & $\mathrm{C}$ & & & 127.6 \\
\hline 33 & 139.1 & $\mathrm{CH}$ & $6.0\left(\mathrm{H}_{33}\right)$ & $20.6\left(\mathrm{C}_{34}\right) ; 166.3\left(\mathrm{C}_{31}\right)$ & 138.8 \\
\hline 34 & 20.6 & $\mathrm{CH}_{3}$ & $1.7\left(\mathrm{H}_{34}\right)$ & $166.3\left(\mathrm{C}_{31}\right) ; 127.6\left(\mathrm{C}_{32}\right) ; 139.1\left(\mathrm{C}_{33}\right)$ & 20.5 \\
\hline 35 & 15.7 & $\mathrm{CH}_{3}$ & $1.9\left(\mathrm{H}_{35}\right)$ & $166.3\left(\mathrm{C}_{31}\right) ; 127.6\left(\mathrm{C}_{32}\right)$ & 15.6 \\
\hline
\end{tabular}

Ultraviolet (UV) spectrum data showed the absorption at 210. At $210 \mathrm{~nm}$ wavelength, show a double bond $(\mathrm{C}=$ C) that was not conjugated, suitable for $\mathrm{C}_{12}$ and $\mathrm{C}_{32}$.

Infrared (IR) spectrum data showed absorption bands at 1457.10 and $1371.38 \mathrm{~cm}^{-1}$, suitable the absorption of gem dimethyl at $\mathrm{C}_{24} ; \mathrm{C}_{23} ; \mathrm{C}_{29}$ and $\mathrm{C}_{30}$ (Figure 1) (Mayanti et al., 2011). Absorption at $3579.32 \mathrm{~cm}^{-1}$ showed the presence of $\mathrm{O}-\mathrm{H}\left(\mathrm{C}_{28}\right)$, supported by the vibration of atom $\mathrm{C}-\mathrm{O}$ at $1034.03 \mathrm{~cm}^{-1}$, and at $1715.66 \mathrm{~cm}^{-1}$ showed the presence $(\mathrm{C}=\mathrm{O})$ carbonil $\left(\mathrm{C}_{3}\right.$ and $\left.\mathrm{C}_{31}\right)$.

${ }^{1} \mathrm{H}-\mathrm{NMR}$ spectrum data $(400 \mathrm{MHz})$ showed nine methyl proton signals at $\delta_{\mathrm{H}}(\mathrm{ppm}) ; 1.2\left(\mathrm{H}_{23}\right) ; 1.1\left(\mathrm{H}_{24}\right) ; 1.0$ $\left(\mathrm{H}_{25}\right) ; 0.8\left(\mathrm{H}_{26}\right) ; 0.9\left(\mathrm{H}_{27}\right) ; 0.9\left(\mathrm{H}_{29}\right) ; 1.0\left(\mathrm{H}_{30}\right) ; 1.7\left(\mathrm{H}_{34}\right)$ and $1.9\left(\mathrm{H}_{35}\right)$, two olefinic proton signals at $\delta_{\mathrm{H}}(\mathrm{ppm}) ; 5.3$ $\left(\mathrm{H}_{12}\right) ; 6.0\left(\mathrm{H}_{33}\right)$ and methine proton signals at $\delta_{\mathrm{H}}(\mathrm{ppm})$; $1.3\left(\mathrm{H}_{5}\right)$ ppm; $5.0\left(\mathrm{H}_{22}\right)$ supported by DEPT 135 data.

${ }^{13} \mathrm{C}$-NMR spectrum data $(100 \mathrm{MHz})$ showed 35 carbon signals. Through DEPT 135 analysis, it's known that from
35 carbon signals were nine methyl carbon (ppm); $\delta \mathrm{c} 26.5$ $\left(\mathrm{C}_{23}\right) ; \delta \mathrm{c} 21.5\left(\mathrm{C}_{24}\right) ; \delta \mathrm{c} 15.1\left(\mathrm{C}_{25}\right) ; \delta \mathrm{c} 16.8\left(\mathrm{C}_{26}\right) ; \delta \mathrm{c} 25.8$ $\left(\mathrm{C}_{27}\right) ; \delta \mathrm{c} 33.7\left(\mathrm{C}_{29}\right) ; \delta \mathrm{c} 26.2\left(\mathrm{C}_{30}\right)$. $\delta \mathrm{c} 20.6\left(\mathrm{C}_{34}\right) ; \delta \mathrm{c} 15.7$ $\left(\mathrm{C}_{35}\right)$. Nine methylene carbon signals were showed at $\delta \mathrm{c}(\mathrm{ppm}) ; \delta \mathrm{c} 39.1\left(\mathrm{C}_{1}\right) ; \delta \mathrm{c} 34.2\left(\mathrm{C}_{2}\right)$. $\delta \mathrm{c} 19.8\left(\mathrm{C}_{6}\right) ; \delta \mathrm{c} 32.2$ $\left(\mathrm{C}_{7}\right) ; \delta \mathrm{c} 23.5\left(\mathrm{C}_{11}\right) ; \delta \mathrm{c} 27.6\left(\mathrm{C}_{15}\right) ; \delta \mathrm{c} 24.2\left(\mathrm{C}_{16}\right) ; \delta \mathrm{c} 46.0$ $\left(\mathrm{C}_{19}\right)$; $\delta \mathrm{c} 37.8\left(\mathrm{C}_{21}\right)$, Six methyne carbon signals were showed at $\delta \mathrm{c}(\mathrm{ppm}) ; \delta \mathrm{c} 55.3\left(\mathrm{C}_{5}\right) ; \delta \mathrm{c} 46.9\left(\mathrm{C}_{9}\right) ; \delta \mathrm{c} 122.6$ $\left(\mathrm{C}_{12}\right) ; \delta \mathrm{c} 38.6\left(\mathrm{C}_{18}\right) ; \delta \mathrm{c} 75.9\left(\mathrm{C}_{22}\right) ; \delta \mathrm{c} 139.1\left(\mathrm{C}_{33}\right)$. Eleven quartener carbon signals were showed at $\delta \mathrm{c}(\mathrm{ppm}) ; \delta \mathrm{c}$ $217.7\left(\mathrm{C}_{3}\right) ; \delta \mathrm{c} 47.5\left(\mathrm{C}_{4}\right) ; \delta \mathrm{c} 39.3\left(\mathrm{C}_{8}\right) ; \delta \mathrm{c} 36.8\left(\mathrm{C}_{10}\right) ; \delta \mathrm{c}$ $143.1\left(\mathrm{C}_{13}\right) ; \delta \mathrm{c} 42.1\left(\mathrm{C}_{14}\right) ; \delta \mathrm{c} 50.6\left(\mathrm{C}_{17}\right) ; \delta \mathrm{c} 30.1\left(\mathrm{C}_{20}\right) ; \delta \mathrm{c}$ $178.4\left(\mathrm{C}_{28}\right) ; \delta \mathrm{c} 166.3\left(\mathrm{C}_{31}\right) ; \delta \mathrm{c} 127.6\left(\mathrm{C}_{32}\right)$. The signal at $\delta \mathrm{c}$ $178.4 \mathrm{ppm}$ showed chemical shift suitable for the carbon carboxylic $\left(\mathrm{C}_{28}\right)$, signal at $\delta \mathrm{c} 166.3 \mathrm{ppm}$ chemical shift suitable for the carbon carboxylic $\left(\mathrm{C}_{31}\right)$ and signal at $\delta \mathrm{c}$ 217.7 ppm showed chemical shift suitable for the carbon carboxylic $\left(\mathrm{C}_{3}\right)$. 


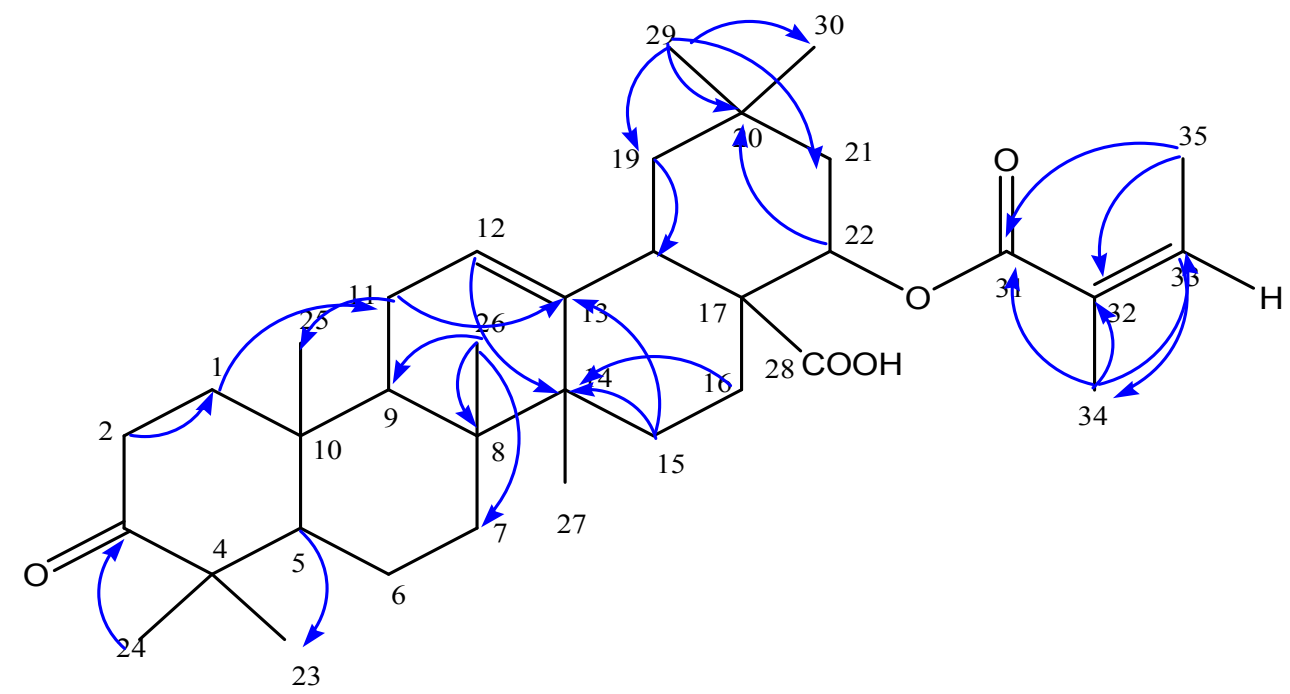

Figure 2. HMBC correlation of Lantadene A

Olefinic carbon signals $\left(\mathrm{C}_{12}\right.$ and $\left.\mathrm{C}_{13}\right)$, which appears at $\delta \mathrm{c} 122.6 \mathrm{ppm}$ and $\delta \mathrm{c} 143.1 \mathrm{ppm})$ and olefinic carbon $\left(\mathrm{C}_{32}\right.$ and $\left.\mathrm{C}_{33}\right)$, which appears at $\delta \mathrm{c} 127.6 \mathrm{ppm}$ and $\delta \mathrm{c} 139.1 \mathrm{ppm}$ supported by HMQC correlation $\mathrm{H}_{12}$ proton $\left(\delta_{\mathrm{H}} 5.3 \mathrm{ppm}\right)$ with $\mathrm{C}_{12}(\delta \mathrm{c} 122.6 \mathrm{ppm})$ and $\mathrm{H}_{33}\left(\delta_{\mathrm{H}} 6.0 \mathrm{ppm}\right)$ with $\mathrm{C}_{33}(\delta \mathrm{c}$ 139.1 ppm).

HMBC correlation spectrum data (Figure 2), showed correlation between $\mathrm{H}_{1}\left(\delta_{\mathrm{H}} 1.5 \mathrm{ppm}\right)$ with $\mathrm{C}_{11}(\delta \mathrm{c} 23.5$ ppm); $\mathrm{H}_{5}\left(\delta_{\mathrm{H}} 1.3 \mathrm{ppm}\right)$ with $\mathrm{C}_{23}(\delta \mathrm{c} 26.5 \mathrm{ppm}) ; \mathrm{H}_{11}\left(\delta_{\mathrm{H}} 1.20\right.$ ppm) with $\mathrm{C}_{13}(\delta \mathrm{c} 143.1 \mathrm{ppm})$ and $\mathrm{C}_{25}(\delta \mathrm{c} 15.7 \mathrm{ppm}) ; \mathrm{H}_{15}$ $\left(\delta_{\mathrm{H}} 1.1 \mathrm{ppm}\right)$ with $\mathrm{C}_{13}(\delta \mathrm{c} 143.1 \mathrm{ppm})$ and $\mathrm{C}_{14}(\delta \mathrm{c} 42.1$ ppm); $\mathrm{H}_{16}\left(\delta_{\mathrm{H}} 0.9 \mathrm{ppm}\right)$ with $\mathrm{C}_{14}(\delta \mathrm{c} 42.1 \mathrm{ppm}) ; \mathrm{H}_{24}\left(\delta_{\mathrm{H}} 1.0\right.$ ppm) with $\mathrm{C}_{3}(\delta \mathrm{c} 217.7 \mathrm{ppm}) ; \mathrm{H}_{26}\left(\delta_{\mathrm{H}} 0.8 \mathrm{ppm}\right)$ with $\mathrm{C}_{7}(\delta \mathrm{c}$ $32.2 \mathrm{ppm}) ; \mathrm{C}_{8}(\delta \mathrm{c} 39.3 \mathrm{ppm})$; and $\mathrm{C}_{9}(\delta \mathrm{c} 46.9 \mathrm{ppm}) ; \mathrm{H}_{29}\left(\delta_{\mathrm{H}}\right.$ $0.9 \mathrm{ppm})$ with $\mathrm{C}_{19}(\delta \mathrm{c} 46.0 \mathrm{ppm}) ; \mathrm{C}_{20}(\delta \mathrm{c} 30.1 \mathrm{ppm}) ; \mathrm{C}_{21}$ ( $\delta \mathrm{c} 37.3 \mathrm{ppm})$; and $\mathrm{C}_{30}(\delta \mathrm{c} 26.2 \mathrm{ppm}) ; \mathrm{H}_{35}\left(\delta_{\mathrm{H}} 1.9 \mathrm{ppm}\right)$ with $\mathrm{C}_{31}(\delta \mathrm{c} 166.3 \mathrm{ppm})$ and $\mathrm{C}_{32}(\delta \mathrm{c} 127.6 \mathrm{ppm})$.

Lantadene A compound has one chiral center at $\mathrm{C}_{8}$, proven by DEPT 135 data. Where at position $\mathrm{C}_{8}$ bound to four different groups at $\mathrm{C}_{26}$ (methyl carbon), $\mathrm{C}_{7}$ (methylene carbon), $\mathrm{C}_{9}$ (methyne carbon) and $\mathrm{C}_{14}$ (quaterner carbon) with each of chemical shift at $\delta \mathrm{c} 16.8\left(\mathrm{C}_{26}\right), \delta \mathrm{c} 32.2\left(\mathrm{C}_{7}\right)$, $\delta c 46.9\left(\mathrm{C}_{9}\right)$ and $\delta c 42.1\left(\mathrm{C}_{14}\right)$, but this paper only compare the similarity of the chemical shift from isolated compound with published compound, not explain the absolute configuration of Lantadene A compound.

\section{Cytotoxic Activity}

The result of the cytotoxic activity Lantadene A isolated compound against shrimp larvae Artemia salina Leach (Table 2). The percentage of mortality of each shrimp larvae Artemia salina Leach in the test solution, calculated using the equation:

$$
\% \text { Mortality }=\frac{\text { total shrimp larvae die }}{\text { total shrimp larvae }} \times 100 \%
$$

From the percentage of mortality value obtained, it is determined the probit value using the probit analysis table. Then, the $\mathrm{LC}_{50}$ value was determined using a regression equation between $\log$ concentration variations with the probit values (Figure 3).

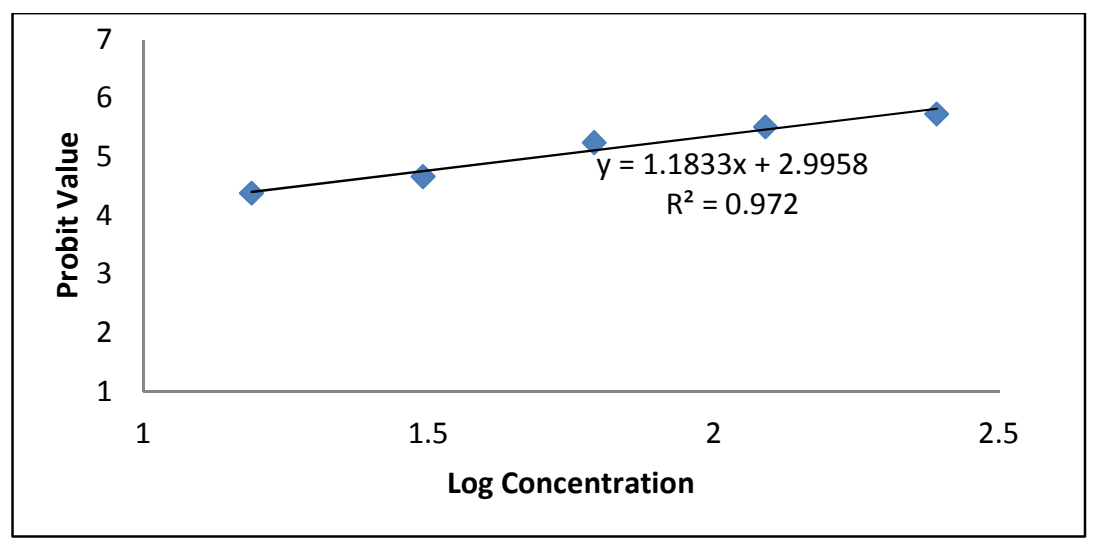

Figure 3. Regression Equation of Log Concentration vs Probit Value of Lantadene A 
Table 2. Data of cytotoxic activity result Lantadene A

\begin{tabular}{ccccccc}
\hline No & $\begin{array}{c}\text { Concentration } \\
(\mu \mathrm{g} / \mathrm{mL})\end{array}$ & $\begin{array}{c}\text { Log } \\
\text { Concentration } \\
(\mathrm{X})\end{array}$ & $\begin{array}{c}\text { Total shrimp } \\
\text { larvae } \\
(\text { tail })\end{array}$ & $\begin{array}{c}\text { Total shrimp } \\
\text { larvae die } \\
(\text { tail })\end{array}$ & $\begin{array}{c}\text { Percentage of } \\
\text { Mortality } \\
(\%)\end{array}$ & $\begin{array}{c}\text { Probit } \\
\text { Value } \\
(\mathrm{Y})\end{array}$ \\
\hline 1. & 15.625 & 1.19 & 30 & 8 & 27 & 4.39 \\
2. & 31.25 & 1.49 & 30 & 11 & 37 & 4.67 \\
3. & 62.5 & 1.79 & 30 & 18 & 60 & 5.25 \\
4. & 125 & 2.09 & 30 & 21 & 70 & 5.52 \\
5. & 250 & 2.39 & 30 & 23 & 77 & 5.74 \\
\hline
\end{tabular}

From regression equation $\mathrm{y}=1.183 \mathrm{x}+2.995$ (Figure 3), obtained the $\mathrm{LC}_{50}$ value of Lantadene $\mathrm{A}$ isolated compound was $48.97 \mu \mathrm{g} / \mathrm{mL}$. Pure compound was declared toxic if has $\mathrm{LC}_{50}$ value $<200 \mu \mathrm{g} / \mathrm{mL}$ (Handayani, Rasyid, Rustini, Zainudin \& Hertiani, 2018). Lantadene A compound was isolated from Lantana camara Linn leaves showed strong cytotoxic activity.

\section{CONCLUSIONS}

The triterpenoid isolated compound from n-hexane fraction of Lantana camara Linn leaves, identified as Lantadene A (22ß-angeloyloxy-3-oxoolean-12-en-28-oicacid). Lantadene A, showed strong cytotoxic activity with $\mathrm{LC}_{50}$ value of $48.97 \mu \mathrm{g} / \mathrm{mL}$.

\section{ACKNOWLEDGMENTS}

Thanks to the Chemistry and Biomolecular Science, Mamoru Koketsu Laboratory for measuring Nuclear Magnetic Resonance (NMR) spectrometer.

\section{REFERENCES}

Abdjul, D.B., Yamazaki, H., Maarisit, W., Rotinsulu, H., Wewengkang, D.S, Sumilat, D.A., Kapojos, M.M., Losung, F., Ukai, K., Namikhosi, M. (2017): Oleanane Triterpenes with Protein Tyrosine Phosphatase 1B Inhibitory Activity from Aerial Parts of Lantana camara Collected in Indonesia and Japan. Phytochemistry, 144, 106-112.

Ediruslan., Manjang, Y., Suryati., Aziz, H. (2015): Structure Elucidation of Brine Shrimp Toxic Compound from Lantana camara L. Leaves. Journal of Chemical and Pharmaceutical Research, 7 (12), 250-255.

Ganjewala, D., Sam, S., Khan, K.H. (2009): Biochemical Compositions and Antibacterial Activities of Lantana camara plants with Yellow, Lavender, Red and White Flowers. EurAsian Journal of BioSciences, 3, 69-77.

Handayani, D., Rasyid, W., Rustini., Zainudin, E.N., Hertiani, T. (2018): Cytotoxic Activity Screening of Fungal Extracts Derived from the West Sumatran Marine Sponge Haliclona fascigera to Several Human Cell Lines: Hela, WiDr, T47D and Vero. Journal of Applied Pharmaceutical Science, 8 (01), 55-58.

Jamal, M., Amir, M., Ali, Z., Mujeeb, M. (2018): A comparative Study for The Extraction Methods and Solvent Selection for Isolation, Quantitative
Estimation and Validation of Ursolic Acid in The Leaves of Lantana camara by HPTLC method. Future Journal of Pharmaceutical Sciences, 4(2), 229-233.

Kazmi, I., Rahman, M., Afzal, M., Gupta, G., Saleem, S., Afzal, O., Shaharyar, M.A., Nautiyal, U., Ahmed, S., Anwar, F. (2012): Anti-diabetic Potential of Ursolic Acid Stearoyl Glucoside: A New Triterpenic Gycosidic Ester from Lantana camara. Fitoterapia, 83, 142-146.

Kumar, R., Katiyar, R., Surender, K., Tarun, K., Singh, V. (2016): Lantana camara: An Alien Weed, Its Impact on Animal Health and Strategies to Control. Journal of Experimental Biology and Agricultural Sciences, 4 (3S), 321-337.

Manu, S., Sharma, P.D., Bansal, M.P., Singh, J. (2007): Lantadene A-Induced Apoptosis in Human Leukemia HL-60 Cells. Indian Journal of Pharmacology, 39(3), 140-144.

Mayanti, T., Tjokonegoro, R., Supratman, U., Mukhtar, M.R., Awang, K., Hadi, A.H. (2011): Antifeedant Triterpenoids from The Seeds and Bark of Lansium domesticum cv Kokossan (Meliaceae). Molecules, 16(4), 2785-2795.

Musa, A.A. (2012): Cytotoxicity Activity and Phytochemical Screening of Cochlospermum tinctorium Perr Ex A. Rich Rhizome. Journal of Applied Pharmaceutical Science, 02 (07), 155-159.

M.Z, Kartika., Suryati., Efdi, M. (2018): A Triterpenoid from The Leaves of Tahi Ayam (Lantana camara Linn). Indonesian Journal of Fundamental and Applied Chemistry, 3 (1), 18-22.

Olowa, L.F., Nuñeza, O.M. (2013): Brine Shrimp Lethality Assay of The Ethanolic Extracts of Three Selected Species of Medicinal Plants from Iligan City, Philippines. International Research Journal of Biological Sciences, 2(11), 74-77.

Passos, J.L., Barbosa, L.C.A., Demuner, A.J., Alvarenga, E.S., Da Silva, C.M., Barreto, R.W. (2012): Chemical Characterization of Volatile Compounds of Lantana camara L. and L. radula Sw. and Their Antifungal Activity. Molecules, 17, 11447-11455.

Pour, B.M., Latha, L.Y., Sashidaran, S. (2011): Cytotoxicity and Oral Acute Toxicity Studies of Lantana camara Leaf Extract. Molecules, 16, 36633674.

Sivasankar, P., Manivasagan, P., Vijayanand, P., Sivakumar, K., Sugesh, S., Poongodi, S., Maharani, 
Molekul, Vol. 14. No. 1, Mei 2019: 31 - 36

V., Vijayalakshmi, S., Balasubramanian, T. (2013): Antibacterial and Brine Shrimp Lethality Effect of Marine Actinobacterium Streptomyces sp. CAS72 Against Human Pathogenic Bacteria. Asian Pacific Journal of Tropical Disease, 3 (4), 286-293.

Suryati., Santoni, A., M.Z, Kartika., Aziz, H., (2016): Antioxidant Activity and Total Phenolic Content of Ethyl Acetate Extract and Fractions of Lantana camara L. Leaf Der Pharma Chemica, 8(8), 92-96.
Vajha, M., Krishna, C.S.R. (2014): Evaluation of Cytotoxicity in Selected Species of Carallum and Boucerosia. Asian Journal of Plant Science and Research, 4 (4), 44-77.

Vyas, N., Argal, A. (2014): Isolation and Characterization of Oleanolic Acid From Roots of Lantana camara. Asian Journal of Pharmaceutical and Clinical Research, 7, 189-191. 